\title{
A PERCEPÇÃO DA IMAGEM DE PREÇO EM SERVIÇOS: UM ESTUDO DE ABORDAGEM QUANTITATIVA SOBRE OS CURSOS DE LATO SENSU DE UMA UNIVERSIDADE
}

\author{
Mateus Panizzon \\ mpanizzo@ucs.br \\ Universidade de Caxias do Sul - Bento Gonçalves, RS / Brasil \\ Deonir De Toni \\ deonirdt@terra.com.br \\ Universidade de Caxias do Sul - Bento Gonçalves, RS / Brasil \\ Fabiano Larentis \\ flarentis@ea.ufrgs.br \\ Universidade de Caxias do Sul - Bento Gonçalves, RS / Brasil \\ Gabriel Sperandio Milan \\ gsmilan@ucs.br \\ Universidade de Caxias do Sul - Bento Gonçalves, RS / Brasil
}

Recebido em 04/04/2012

Aprovado em 14/03/2013

Disponibilizado em 01/12/2013

Avaliado pelo sistema double blind review

Revista Eletrônica de Administração

Editor: Luís Felipe Nascimento

ISSN 1413-2311 (versão on-line)

Editada pela Escola de Administração da Universidade Federal do Rio Grande do Sul.

Periodicidade: Quadrimestral

Sistema requerido: Adobe Acrobat Reader.

\section{RESUMO}

A imagem de preço pode ser definida como uma variável latente e multidimensional que consiste numa convicção subjetiva e emocional associada a fatores relacionados com o preço de um produto ou serviço (ZIELKE, 2006; 2011). Assim, este estudo identifica que a imagem de preço de serviço pode ser mensurada a partir de quatro dimensões: Dimensão Funcional, Valor Percebido, Nível de Preço e Dimensão Emocional. A partir destes fatores este trabalho buscou testar e validar uma escala de imagem de preço de serviço de modo a identificar a influência destas dimensões sobre as intenções de recompra. Tal escala foi aplicada a uma amostra de 159 estudantes dos cursos de pós-graduação lato sensu de uma Universidade privada. Os resultados apontam que esta escala apresenta uma boa confiabilidade, validade e que as dimensões funcionais, relacionadas aos aspectos da infra-estrutura, ao corpo docente e a qualidade, e a dimensão de valor percebido, relacionada à percepção de custo versus benefícios são as que mais influenciam na intenção de recompra dos estudantes. Ainda, as análises multivariadas de regressão, ANOVA e cluster apontaram diferenças significativas na percepção das dimensões e na intenção de recompra entre os estudantes no início, meio e final do curso, o que reflete a natureza e a complexidade deste constructo, uma vez que está associado à experiência de consumo.

REAd | Porto Alegre - Edição 76 - N 3 - setembro/dezembro 2013 - p. 646-674 
A percepção da imagem de preço em serviços: um estudo de abordagem quantitativa sobre os cursos de lato sensu de uma universidade

Palavras-Chave: Imagem de Preço; Preço; Comportamento do Consumidor.

\title{
THE PRICE IMAGE PERCEPTION IN SERVICES: A QUANTITATIVE RESEARCH AT POSTGRADUATE UNIVERSITY COURSES
}

\begin{abstract}
The concept of price image can be defined as a multidimensional latent variable that consists in a subjective and emotional conviction, which is associated to several factors related to a product or service price (ZIELKE, 2006; 2011). This study identifies that the price image of a service can be measured by four dimensions: Functional Dimension, Perceived Value, Price Level and Emotional Dimension. Grounded in these factors, this paper seeks to test and validate an service price image scale and investigate the relationship of this variables with the re-buy intention. The scale was applied to a sample of 159 postgraduate students of a private University. The results point that this scale presented a good degree of reliability and validity. The findings showed that the functional dimensional, related to infra-structure, university faculty, and quality, and the perceived value dimension, related to perception of cost versus benefits, have the most influence in the re-buy intention. Yet, multivariate analysis like regression, ANOVA, and cluster pointed significant differences in the perception of this dimensions and the re-buy intention of this students in the beginning, middle and final stage of the course, which suggest a complex and dynamic nature of this construct, due your association to consumption experience.
\end{abstract}

Keywords: Price Image; Price; Consumer Behavior.

\section{INTRODUÇÃO}

De acordo com Zielke (2010), o preço é uma variável dominante nos aspectos mercadológicos. Portanto, o domínio do melhor preço em relação aos concorrentes é uma tarefa crucial para os gestores das organizações que atuam com a elaboração das estratégias de precificação. Contudo, pouca literatura tem discutido acerca da percepção do preço pelos clientes, e como este constructo de fato interfere nas complexas imagens que eles têm dos produtos ou da própria organização (imagem de preço). Em síntese, para o autor, a imagem de preço não é uma simples tradução do preço atual cobrado pela empresa. O preço envolve processos subjetivos, tais como o preço percebido, que pode ser influenciado inclusive pela imagem da organização como um todo, e também por sua publicidade, por seu tamanho, ou bem como pelo seu nível dos serviços de suporte prestados. Neste sentido, e conforme apresentado por Bolton, Warlop e Alba (2003), a pesquisa sobre o fator preço, e principalmente, o preço justo percebido, necessita de maior aprofundamento.

REAd | Porto Alegre - Edição 76 - N 3 - setembro/dezembro 2013 - p. 646-674 
O conceito de imagem de preço, conforme Zielke (2010) pode ser definido como uma variável latente multidimensional que consiste em crenças e sentimentos subjetivos (armazenados na memória do consumidor), sobre as atividades de preço de uma empresa, loja ou categoria. Baseado nestas crenças e sentimentos os consumidores podem derivar uma avaliação geral da política de preço que pode ser rotulada de "atitude de preço", e que está fortemente associada ao comportamento de compra. Este conceito pode ser percebido, por exemplo, em marcas de luxo. O consumidor sabe, antes mesmo de entrar no estabelecimento, que faixa de preço os produtos possuem, e que tipo de postura de compra é esperado do cliente dentro desta loja.

O objetivo deste estudo é validar as dimensões que podem configurar a imagem de preço de serviço e seus impactos sobre as intenções de recompra. Para tanto, a partir da adaptação da escala de Zielke (2010) de imagem de preço para varejo e do estudo qualitativo de Schuler et al. (2010) de imagem de preço para serviços, identificou-se que a imagem de preço de serviço pode ser configurada a partir de elementos funcionais, de nível de preço, de valor percebido e de fatores emocionais. Assim, foi proposta uma escala adaptada principalmente dos estudos de Zielke $(2006,2010)$ e aplicada junto a uma amostra de 159 estudantes dos cursos de lato sensu de uma Universidade privada. Os resultados apontam que esta escala apresenta uma boa confiabilidade, validade e que as dimensões funcionais, relacionadas aos aspectos da infraestrutura, ao corpo docente e a qualidade, e a dimensão de valor percebido, relacionada à percepção de custo versus benefícios são as que mais influenciam na intenção de recompra dos estudantes. A contribuição desta pesquisa reflete-se no fato de que enquanto a escala de Zielke (2010) foi desenvolvida para o contexto de varejo, esta escala se propõe a mensurar o contexto de serviços educacionais, por se tratar de uma lacuna de pesquisa. Os resultados refletem, portanto, que dada a emergência de outras dimensões envolvidas, torna-se apropriado observar este contexto de aplicação no aprimoramento do corpus teórico da Imagem de Preço em futuros estudos.

Sendo assim, o artigo está estruturado da seguinte forma: o referencial teórico, com enfoque em impacto do preço nas decisões, imagem de preço e intenção de compra e dimensões da imagem de preço. O objetivo deste referencial é, no primeiro momento, resgatar as bases da influência do preço como variável moderadora das decisões de compra, convergindo para o detalhamento das dimensões e indicadores utilizados para mensurar o fenômeno de Imagem de Preço no varejo, originalmente validado por Ziekle (2010) e propostos para serviços em Schuler et al. (2010). Em sequiência, descreve-se o método 
A percepção da imagem de preço em serviços: um estudo de abordagem quantitativa sobre os cursos de lato sensu de uma universidade

quantitativo de pesquisa; a análise dos resultados, buscando evidenciar a validação da escala e a compreensão da relação entre a imagem de preço e intenção de recompra, com base nas técnicas de regressão múltipla, análise de clusters e análise de variância e considerações finais.

\section{REFERENCIAL TEÓRICO}

Estudos de imagem de preço ainda são recentes e tem sido principalmente desenvolvidos no contexto do varejo (ZIELKE E TOPOROWSKI, 2012; VIEIRA E MATOS, 2012; ZIELKE, 2010, ZIELKE, 2008, JANISESWKI e LICHESTEIN, 1999), sendo que a partir de Zielke (2008), que se passou a ter um constructo validado de imagem de preço.

Outro aspecto é a carência de estudos sobre a influência do preço no contexto que se refere a serviços, e no que tange ao contexto das Instituições de Ensino Superior, que se constitui alvo do recorte deste estudo, o que demonstra ser uma lacuna de pesquisa. Com base em consulta nos dois principais periódicos que publicam trabalhos com enfoque mercadológico e gestão nas Universidades, o Journal of Marketing for Higher Education e o Journal of Higher Education Policy and Management, foram localizados apenas cinco artigos que tratavam de analisar o efeito do preço e descontos na percepção dos alunos (ODUOZA, 2009; COATS, 2008; DEMORANVILLE e O`DONNEL, 2008; QUIGLEY JR, BINGHAM, NOTARANTONIO, MURRAY, 2000; LITTLE, O`TOOLE e WETZEL, 1997).

Essencialmente, os resultados destes estudos apontam a significância deste atributo, e das suas relações em termos de valor percebido, de resposta de compra de crédito dos alunos e das variações de elasticidade, da associação entre preço e qualidade. Também são identificados estudos de como as IES são avaliadas em termos de seu posicionamento de alto preço e baixo preço. Tais estudos embasam políticas e decisões estratégicas que Instituições podem adotar ou não em termos de preço. No estudo de Quigley Jr. et al. (2000), por exemplo, concluiu-se que a prática de descontos pouco influenciava na contratação do serviço desejado. Gerencialmente, a adoção desta prática sem uma maior fundamentação poderá gerar para a organização não só prejuízos financeiros, mas desvalorização da imagem de preço que o aluno tem daquela Instituição, o que justifica maior compreensão e pesquisas neste tema.

Neste aspecto, e considerando a escassez de estudos desta natureza no contexto do ensino superior privado brasileiro, esta pesquisa buscou fundamentar sua base na questão do 
preço e seus impactos nas decisões estratégicas, na imagem de preço e intenção de compra e nas dimensões que compõem o constructo imagem de preço.

\subsection{Preço e seus impactos nas decisões estratégicas}

Tradicionalmente empresas e pesquisadores têm dado pouca atenção no desenvolvimento de novas abordagens para auxiliar no processo de tomada de decisão no apreçamento dos produtos ou serviços. Assim, as estratégias de precificação não podem ser tratadas de forma isolada da estratégia global da organização, visto que seus impactos são muito fortes na sua sustentabilidade e crescimento (AVLONITIS; INDOUNAS, 2006). Empresas que têm sucesso nas decisões lucrativas de preço têm realizado uma abordagem proativa no seu apreçamento. Estas empresas buscam considerar os efeitos de uma decisão de preço na percepção dos compradores e como estes compradores desenvolvem uma percepção de valor.

Segundo Monroe (1990), há dois pré-requisitos para tornar o preço proativo um sucesso. Primeiro, é necessário entender como o preço trabalha, seus impactos nos fornecedores, pessoal de venda, distribuidores, competidores e clientes. Segundo, é essencial identificar como o cliente percebe o preço e suas mudanças. Dentro desta lógica uma visão mais externa no apreçamento do produto se faz necessária para que a empresa obtenha melhores resultados.

Desse modo, o paradigma do mero repasse dos custos, somado ao lucro para a formação do preço de venda, necessita ser analisado a partir das novas contingências mercadológicas. Ou seja, é imprescindível considerar, e/ou adotar, estratégias que buscam uma visão não só interna, de dentro para fora da empresa, mas também externa, de fora para dentro. Buscar uma análise mais profunda do mercado, verificando o que os clientes atuais e potenciais estão dispostos a pagar por produtos ou serviços das empresas, compreendendo o que é valor na percepção dos clientes, quais suas imagens com relação ao preço, desponta como alternativas para melhorar o desempenho das organizações (BERNARDI, 1996).

Segundo Ramos, Maya e Bornia (2005), a maneira mais rápida e mais eficaz para que uma empresa maximize seu lucro é começar fixando o preço corretamente. Além disso, o preço tem um impacto muito forte sobre o volume de vendas e sobre a participação de mercado (market share). Sua influência temporal é mais rápida, até imediata, em comparação a outros elementos do composto mercadológico, e pode ser modificada com relativa rapidez.

REAd | Porto Alegre - Edição 76 - N 3 - setembro/dezembro 2013 - p. 646-674 
A percepção da imagem de preço em serviços: um estudo de abordagem quantitativa sobre os cursos de lato sensu de uma universidade

Sendo assim, a forma pela qual as empresas definem seus preços não influencia apenas o comportamento da demanda, mas o modo como os compradores utilizam o produto e/ou o serviço, podendo impactar positivamente no estabelecimento, na manutenção e na ampliação de relacionamentos duradouros entre as partes, ou seja, entre comprador e vendedor (GOURVILLE; SOMAN, 2002). Dovaliene e Virvilaite (2008) indicam que a satisfação com o preço, a aceitabilidade do preço auxilia muito na manutenção do relacionamento. Da mesma forma a aceitabilidade dos preços podem ser um dos fatores para o aumento da lealdade, ou seja, a longevidade do relacionamento (VIRVILAITE et al., 2009).

Geralmente, costuma-se tratar a precificação como a estratégia mais simples dentro do composto de marketing. Resultados provenientes de estudos realizados por Simon (1992), Souza et al. (2005) e Souza (2006) indicam que muitas empresas determinam seus preços com base na intuição, em paradigmas vigentes e na experiência de mercado dos gestores. Não há, em muitos casos, a preocupação com um sistema de informações que dê suporte, em um nível adequado, ao processo de tomada de decisões, seja na estimativa dos custos, seja na formação dos preços. A capacidade de coletar, armazenar, processar, disponibilizar e acessar informações oriundas dos ambientes interno e externo constitui um recurso imperioso no que se refere ao suporte às decisões de preço de maneira confiável (KELLER; KOTLER, 2006).

Do ponto de vista gerencial, práticas de apreçamento negociáveis que dão poder para a área de vendas fixarem os preços de maneira tática, ou seja, negócio a negócio, ensinam o pessoal de vendas a fechar negócios pela via fácil, preço, em vez de elevar a disposição de o cliente pagar por meio de uma venda de valor (HOLDEN; NAGLE, 2003). Nesse sentido, desenvolver uma imagem de valor de um produto é um desafio de muitas organizações. Para isso, os vendedores precisam reaprender a comunicar valor em vez de negociar descontos. Comunicar valor não significa que os preços são inegociáveis, mas que os preços podem ser variáveis em condições de volumes maiores, contratos de longo prazo, compras empacotadas, etc.

O preço de uma organização também informa ao mercado o posicionamento de valor pretendido pela empresa de seu produto ou marca (KELLER; KOTLER, 2006). No entanto, como afirma Kearney (2004), é preciso definir o valor a partir do ponto de vista do comprador, e não do vendedor, e para isso é preciso entender os benefícios que o comprador de fato percebe. Dessa forma, Nagle e Holden (2003) propõem três razões pela qual essa análise deve ser feita. Primeiro, ela pode identificar segmentos de mercado com probabilidade de apresentar diferentes sensibilidades a preço. Segundo, pode indicar a faixa de preços dentro

REAd | Porto Alegre - Edição 76 - N 3 - setembro/dezembro 2013 - p. 646-674 
do qual a empresa deverá apreçar seus produtos. Terceiro, os gerentes precisam analisar a sensibilidade a preço do comprador para determinar como efetivamente influenciá-lo.

De forma estrita, o preço representa a soma de dinheiro que deve ser gasto, sendo que um preço alto pode afetar negativamente uma probabilidade de compra. No entanto o preço é um estímulo complexo que não tem apenas um papel negativo, mas também um papel positivo, tal como uma dica de qualidade (LICHTENSTEIN et al., 1993). Assim, empresas proativas na formação de preço devem aprender como seus clientes percebem, qual imagem eles tem do preço e como estas imagens influenciam sua percepção de valor e intenção de compra. Pois segundo Monroe (1990, p. 84), a relação entre preço e a percepção de valor do cliente é que determina as decisões de compra.

A formação de qualquer estratégia de apreçamento deve começar com uma análise das imagens de preço que os consumidores têm e as diferentes formas de valor que ele representa para o indivíduo. Identificar as percepções de valor e da sensibilidade a preço auxilia na definição de estratégias de preço focadas na satisfação do comprador e na lucratividade do vendedor.

\subsection{Imagem de preço e intenção de compra}

Numa perspectiva mercadológica, Barich e Kotler (1991) apresentam quatro tipos de imagem: a da marca (como as pessoas percebem uma marca particular em relação às suas concorrentes); a dos produtos (como as pessoas vêem uma determinada categoria de produtos); a de marketing (a forma como é vista a qualidade da oferta e o mix de marketing da organização) e a imagem corporativa (como as pessoas percebem a empresa como um todo). Já Stern et al. (2001) confirmam três diferentes, mas relacionados tipos de imagens: imagens da marca e/ou produto; imagem da corporação; e imagem do estabelecimento comercial. O que se observa que, apesar da importância que a dimensão preço assume no comportamento de compra, há poucos estudos que buscam aprofundar a imagem de preço e seus impactos nas intenções de compra.

Uma questão que intriga muitos pesquisadores é: “como o preço afeta as decisões de compra?" O sucesso de uma formação de preços proativo deve ser consistente com o valor percebido pelo cliente. Para entender o valor percebido pelo cliente é importante primeiro entender como as pessoas formam as percepções de preço e como o processo perceptual influencia suas imagens.

REAd | Porto Alegre - Edição 76 - N 3 - setembro/dezembro 2013 - p. 646-674 
A percepção da imagem de preço em serviços: um estudo de abordagem quantitativa sobre os cursos de lato sensu de uma universidade

O preço foi durante muito tempo e ainda continua a tratado por grande parte das empresas como apenas uma questão financeira. No entanto, o que se percebe que o preço está associado a várias dimensões da percepção humana. O estudo da imagem de preço é um tema pouco abordado na literatura (ZIELKE, 2006, 2010), mas que merece uma atenção tanto da academia quanto do meio gerencial, por sua influência nas decisões de compra (MONROE; 1990; HOLDEN; NAGEL, 2003).

A percepção pelo consumidor de um preço deriva de sua interpretação das diferenças de preço e de sua interpretação da oferta. Assim, os consumidores tomam suas decisões em dois passos: primeiro ele julga o valor da oferta e depois ele decide se faz ou não a compra. Assim, a dificuldade em entender, planejar e implementar políticas de preço não deve ser subestimada. O desenvolvimento de um relacionamento de longo prazo com o cliente através do ajuste dos preços a partir do entendimento de suas necessidades é algo fundamental para desenvolver uma empresa orientada para o mercado (AVLONITIS; INDOUNAS 2006).

A precificação faz com que o consumidor elabore associações com o produto, além de sua percepção do custo de aquisição (PAIXÃO et al., 2006). Nesse sentido, observa-se que o preço assume diferentes associações que vão depender do grau de valor atribuído ao produto ou serviço. Para Zielke (2006; 2010), a imagem do preço pode ser definida como uma variável latente multidimensional que consiste de convicções subjetivas e sentimentos (armazenadas na memória dos clientes) em relação aos preços. Baseado nestas convicções e sentimentos, o consumidor pode fazer uma avaliação geral da política de preço do objeto. Assim, a imagem de preço é considerada um dos principais fatores que influenciam a decisão de compra do consumidor, pois muitos consumidores usam a informação do preço, como unidade de mensuração de valor (BOLTON et al., 2008; ZEITHAML, 1988).

Sendo a imagem um processo pelo qual as pessoas selecionam, organizam e interpretam as informações para formar um significado do objeto, a imagem de preço está muito relacionada a um julgamento do consumidor sobre a média de preço de um produto ou serviço em comparação com algum competidor deste produto ou serviço (MUNNUKKA, 2006). Assim, é necessário que os gestores entendam quanto à percepção do comprador sobre preço, para estabelecer e comunicar suas estratégias de preço (CAMPO; YAGÛE, 2007).

O estudo de Siems et al. (2008) a partir do modelo de satisfação e lealdade de Fornell et al. (1996) inclui o constructo percepção de preço como um influenciador da satisfação e da lealdade. Seus achados indicam que a percepção de preço não influenciou significativamente no construto satisfação, no entanto a percepção de preço foi significativa no construto 
lealdade. Mesmo com estes achados, que a partir de algumas limitações de sua coleta, os autores reafirmam que os efeitos da satisfação do preço possuem uma relação positiva com a satisfação geral do consumidor e consequentemente na lealdade do consumidor.

Estudos em estabelecimentos comerciais demonstram que a imagem do preço tem um impacto direto no que o consumidor espera pagar. Os experimentos de Hamiliton e Chernev (2010) identificam que lojas com um posicionamento requintado têm uma imagem de preços altos quando os participantes tinham um foco apenas investigativo de compra. De modo particular, pesquisas mostram que o local onde o consumidor compra tem um forte impacto em como o consumidor percebe o preço (HAMILTON; CHERNEV, 2010).

Há muitos exemplos e diferenças de comportamento em cada situação de preço. Por exemplo, Winer (1985) identificou que as expectativas futuras de preço têm um papel significante nas decisões de compra. Consumidores que tem expectativas de um preço futuro maior são encorajados a comprar antes que os consumidores que tem como expectativa um preço futuro menor, os quais são mais prováveis a esperar suas compras (TSIROS; HARDESTY, 2010). Outros estudos de Peine et al. (2009) identificam que os consumidores que percebem uma oferta alvo com mais valor e justa também apresentam maior desejo de comentar palavras positivas e uma maior intenção de compra. Já uma percepção de preço negativa está mais associada a um comportamento de compra passivo (PEINE et al., 2009).

O preço não tem apenas uma função "negativa", ou de valor pago, ou sacrifício, mas também serve como dica de qualidade ou benefício. Assim o preço tem também um papel "positivo", sendo que o preço alto afeta positivamente a probabilidade de compra (LICHTENSTEIN et al., 1993). Consistente com essa visão os autores indicam cinco construtos que representam o papel negativo do preço e dois que representam o papel positivo do preço. Como papel negativo tem-se: a) consciência de valor: definido como uma preocupação com o preço pago com relação à qualidade recebida; b) consciência do preço: é o grau no qual o consumidor foca exclusivamente em pagar um menor preço; c) propensão a cupons: refere-se ao aumento da propensão à compra em função das formas dos cupons que afetam positivamente na avaliação de compra; d) propensão à venda: refere-se ao aumento da propensão para responder a uma oferta de compra em função da forma de venda na qual o preço é apresentado; e) conhecedor de preço: definido o grau no qual o indivíduo é uma fonte de informação de preço para muitos tipos de produtos e locais para comprar pelo menor preço e sabe muito sobre as informações dos preços de mercado. Como papel positivo o preço tem dois constructos: a) esquema de preço-qualidade: refere-se que o preço é uma dica que está 
A percepção da imagem de preço em serviços: um estudo de abordagem quantitativa sobre os cursos de lato sensu de uma universidade

positivamente relacionada com o nível de qualidade do produto; b) prestígio: refere-se a uma favorável percepção do preço como uma dica de status. Dessa forma, percebe-se claramente a heterogeneidade dos clientes em termos de atenção e reação com relação aos preços ofertados.

Os estudos de Campo e Yague (2007) indicam que a consciência do preço difere entre grupos demográficos, sendo que o maior nível de consciência está nos consumidores que são mulheres, casadas, mais velhas e não trabalham fora de casa. $\mathrm{O}$ artigo também identifica que a formação da percepção do preço como valor monetário difere de acordo com as características individuais. Assim, consumidores com menor renda usam mais o preço armazenado na sua memória para formar suas percepções de preço (CAMPO; YAGÜE, 2007).

Os consumidores compram a partir da escolha de um conjunto de produtos que maximize sua utilidade, dado o preço de outros produtos e dado o preço que eles podem dispor a pagar (WERTENBROCH et. al., 2007). Nesse sentido, o conceito de imagem de preço vai além de ser simplesmente uma dica extrínseca ou racional do produto como colocado por Zeithaml (1988). Ela pode congregar também sensações, emoções, percepções, conceitos, informações, idéias, suposições e expectativas, sendo que a constituição de uma imagem depende da forma específica pela qual um indivíduo ou grupo trata as informações colhidas do contato com o objeto da imagem (DOBNI; ZINKHAN, 1990; STERN et al., 2001; SHAPIRO, 1968; POIESZ, 1989). Este achado corrabora com as pesquisas de Peine et al. (2009) em que identifica que as respostas ao comportamento de compra para uma informação de preço são dirigidas tanto por fatores cognitivos quanto afetivos.

Desta forma, identifica-se que a imagem de preço pode ser influenciada pelos seguintes aspectos: a) pela percepção individual do consumidor de um conjunto de atributos salientes contidos no objeto; b) pela importância ou envolvimento que o consumidor dá ao objeto; c) pelas influências sócio-culturais do ambiente em que vive; d) pelas diversas ações mercadológicas realizadas pelas organizações a fim de influenciar suas decisões de compra (DE TONI, 2005).

\subsection{Dimensões da imagem de preço}

Para Zielke (2010), a multidimensionalidade da questão preço pode ser transcrita em termos de cinco dimensões, as quais levariam a uma intenção de compra.

A primeira dimensão, percepção do nível de preço (price level) considera a percepção dos preços sem levar em conta diferenças de qualidade. Nesta dimensão ocorrem os processos

REAd | Porto Alegre - Edição 76 - N 3 - setembro/dezembro 2013 - p. 646-674 
de ancoragem, na qual o consumidor baseia-se em referências para decidir qual é o produto com a melhor relação custo-benefício (ARIELY, 2008).

A segunda dimensão, valor do dinheiro (value for money) trata do trade-off entre os sacrifícios e utilidades derivadas do produto. Zeithmal) aponta que o valor é a avaliação global do consumidor da utilidade de um produto baseado na percepção do que é recebido e do que é dado, e leva em conta fatores como: preço monetário percebido e não percebido, sacrifício monetário e não monetário, abstrações, e atributos intrínsecos e extrínsecos. Do ponto de vista estratégico, entender que valor os clientes dão aos produtos e serviços da empresa é fundamental pois a entrega de valor superior (percebido) está positivamente relacionada ao desempenho financeiro da empresa e consequentemente ao aumento de valor ao acionista (LARENTIS; GASTAL, 2004).

A terceira dimensão, perceptibilidade de preço (price percebility) indica o quão facilmente o consumidor pode perceber e analisar os preços dos produtos, e está associado aos aspectos informacionais visuais.

A quarta dimensão, processabilidade de preço (price processability) indica o quão fácil é para o cliente processar este preço, principalmente em relação à comparação com produtos similares.

A quinta dimensão, avaliação da certeza do preço (evaluation certanty), refere-se à quão facilmente o consumidor consegue julgar se um preço é justo para um determinado produto ou pelo julgamento do estabelecimento como um todo. Monroe (2003) aponta que o preço percebido é fundamental para a percepção do valor, envolvendo os benefícios e sacrifícios percebidos. Em síntese, um preço alto poderá aumentar o benefício percebido caso sinalize alta qualidade, mas poderá ter o efeito reverso, se considerado de forma inexplicável abusivo; a isso esta percepção de preço e valor é chamada de custo-benefício, a partir do preço percebido de determinada oferta (MAYER; MARIANO; ANDRADE, 2009).

Schuler et al. (2010), ao desenvolverem uma investigação qualitativa sobre Imagem de Preço no contexto de serviços sobre a mesma população desta pesquisa, observaram que imagem de preço evidenciou aspectos mais positivos do que negativos, na visão dos alunos, mas não demonstrou uma uniformidade, o que sugere que esta imagem é difusa para o cliente final. Neste sentido, para os autores, ainda que um curso de lato sensu seja constituído de atributos racionais e simbólicos conforme observado nas categorias emergentes, e que tais atributos tenham convergência com o modelo de Zielke (2010), não se pôde verificar que a imagem de preço dos cursos de lato sensu desta instituição estivesse claramente definida.

REAd | Porto Alegre - Edição 76 - Nº 3 - setembro/dezembro 2013 - p. 646-674 
A percepção da imagem de preço em serviços: um estudo de abordagem quantitativa sobre os cursos de lato sensu de uma universidade

No estudo de Schuler et al. (2010) são apresentados doze dimensões que compõem a imagem de preço. Identifica-se que as dimensões funcionais e emocionais não foram devidamente contempladas pelo estudo de Zielke (2010), mesmo porque tal estudo teve a intenção de mensurar a imagem de preço de varejo.

A dimensão funcional é a eficiência na qual o produto ou serviço exerce sua função primária e direta. Resume-se no valor das características do produto ou do serviço para satisfazer a necessidade que motivou a compra, no valor da facilitação dos processos de tomada de decisão que o produto fornece. $\mathrm{O}$ valor funcional está relacionado à utilidade percebida a partir de sua habilidade de executar seus propósitos funcionais, utilitários e físicos (SHETH; NEWMAN; GROSS, 1991).

A dimensão Emocional pode ser definida como a capacidade de um produto ou serviço de despertar sentimentos e emoções desejadas e de promover prazer, seja sensorial (beleza, perfume, sonoridade, sabor, sensações táteis agradáveis), seja de bem estar (segurança, contentamento). Assim, a dimensão emocional é derivada de um sentimento ou estado afetivo que pode impactar em diferentes situações de escolha de um objeto (SHETH; NEWMAN; GROSS, 1991).

Assim, a partir da revisão da literatura baseada no modelo de Zielke (2010) e no estudo exploratório de Schuler et al. (2010), identificou-se que a imagem de preço de pósgraduação lato sensu pode ser composta de quatro dimensões, sendo elas: a) níveis de preço, que indica a percepção de preço enquanto alto ou baixo; b) funcional, que indica aspectos relacionados à atributos de infraestrutura e qualidade do curso; c) emocional, cuja manifestação pode estar relacionada à percepção de injustiça e insatisfação; d) o valor percebido, que indica o quanto o custo-benefício é percebido pelos estudantes no antes, no início e durante o curso.

\section{MÉTODO DE PESQUISA}

Para este estudo de caráter exploratório e descritivo, e de abordagem quantitativa, utilizou-se como estratégia de pesquisa a survey (HAIR, 2005).

O questionário foi elaborado com questões fechadas, com base no modelo de Zielke (2010). As dimensões consistiram de 26 itens no total, cuja escala de concordância tipo likert de 7 pontos foi utilizada, variando de "discordo totalmente até concordo totalmente. Originalmente, a escala do autor previa as seguintes dimensões: (i) Percepção do Nível de 
Preço; (ii) Valor do Dinheiro; (iii) Perceptibilidade do Preço; (iv) Processabilidade do Preço; (v) Avaliação da Certeza do Preço; (vi) Intenção de compra.

Entretanto, tendo em vista que a escala original de Zielke (2010) foi validada no contexto do varejo, foi verificado que determinadas dimensões não se aplicavam ao contexto dos serviços, o qual é escopo deste estudo. Por exemplo, as questões referentes à perceptibilidade do preço físico (comparar a diferença de preço entre os produtos de uma loja, a partir de cartazes, etiquetas) não se aplicavam ao contexto de serviços dos cursos de lato sensu. Neste caso não há o mesmo produto de diferentes marcas dentro do "estabelecimento IES", o que implicaria na comparação para o consumidor, mas de diferentes cursos da mesma marca. Neste sentido, procederem-se com as devidas alterações para adaptação ao contexto de serviços, orientando-se também pelas dimensões emergidas no estudo de Schuler et al. (2010), aplicado a esta população sendo que a escala final ficou configurada de acordo com o Quadro 1. O instrumento sofreu tradução reversa, validação nomológica e pré-teste.

Quadro 1 - Itens da Escala de Imagem de Preço.

\begin{tabular}{|c|c|c|c|}
\hline Construto & $\mathbf{N}^{\mathbf{0}}$ & Variáveis & Adaptado de: \\
\hline \multirow{4}{*}{$\begin{array}{l}\text { Intenção } \\
\text { de } \\
\text { Recompra }\end{array}$} & 12 & Costumo recomendar pós-graduação da INSTITUIÇÃO para outras pessoas & $\begin{array}{l}\text { Sweeney et al. } \\
(2001)\end{array}$ \\
\hline & 13 & $\begin{array}{l}\text { Em função do preço, eu deveria investir mais em outras pós-graduações na } \\
\text { INSTITUIÇÃO }\end{array}$ & $\begin{array}{l}\text { Sweeney et al. } \\
(2001)\end{array}$ \\
\hline & 15 & Eu deveria estudar na Instituição o mais frequentemente possível & Zielke (2010) \\
\hline & 17 & $\begin{array}{l}\text { Eu deveria considerar os cursos de pós-graduação da Instituição para meus } \\
\text { futuros estudos }\end{array}$ & Zielke (2010) \\
\hline \multirow{6}{*}{$\begin{array}{l}\text { Dimensão } \\
\text { Funcional }\end{array}$} & 20 & $\begin{array}{l}\text { Se eu avaliar os atributos do curso de pós-graduação da Instituição, tal } \\
\text { como infraestrutura, o preço é adequado }\end{array}$ & $\begin{array}{l}\text { Schuler } \text { et al. } \\
(2010)\end{array}$ \\
\hline & 21 & $\begin{array}{l}\text { Se eu avaliar os atributos do curso de pós-graduação da INSTITUIÇÃO, } \\
\text { tal como o corpo-docente, o preço é adequado }\end{array}$ & $\begin{array}{l}\text { Schuler et al. } \\
(2010)\end{array}$ \\
\hline & 19 & $\begin{array}{l}\text { Em relação ao nível de qualidade do curso de pós-graduação da } \\
\text { INSTITUIÇÃO, o preço é bastante adequado }\end{array}$ & $\begin{array}{l}\text { Schuler et al. } \\
(2010)\end{array}$ \\
\hline & 24 & $\begin{array}{l}\text { Se eu avaliar os atributos da INSTITUIÇÃO, tal como as disciplinas } \\
\text { oferecidas, o preço é adequado }\end{array}$ & $\begin{array}{l}\text { Schuler et al. } \\
(2010)\end{array}$ \\
\hline & 22 & $\begin{array}{l}\text { Se eu avaliar os atributos da INSTITUIÇÃO, tal como a biblioteca,o preço } \\
\text { é adequado }\end{array}$ & $\begin{array}{l}\text { Schuler et al. } \\
\text { (2010) }\end{array}$ \\
\hline & 25 & $\begin{array}{l}\text { Se eu avaliar a localização e a acessibilidade da INSTITUIÇÃO, o preço é } \\
\text { adequado }\end{array}$ & $\begin{array}{l}\text { Schuler et al. } \\
(2010)\end{array}$ \\
\hline \multirow{5}{*}{$\begin{array}{l}\text { Valor } \\
\text { Percebido }\end{array}$} & 5 & Este curso de pós-graduação vale o que eu pago por ele & $\begin{array}{l}\text { Zielke (2010), } \\
\text { Zeithalm (1988) }\end{array}$ \\
\hline & 6 & $\begin{array}{l}\text { Comparada a outras instituições, o custo-benefício é muito bom na } \\
\text { Instituição }\end{array}$ & Zielke (2010) \\
\hline & 29 & $\begin{array}{l}\text { Minha percepção de custo-benefício era melhor antes do início das aulas } \\
\text { (Escala Reversa, ajustada posteriormente na análise) }\end{array}$ & $\begin{array}{l}\text { Zielke (2010), } \\
\text { Zeithalm (1988) }\end{array}$ \\
\hline & 30 & $\begin{array}{l}\text { Minha percepção de custo-benefício piorou logo após o início das aulas } \\
\text { (Escala Reversa, ajustada posteriormente na análise) }\end{array}$ & $\begin{array}{l}\text { Zielke (2010), } \\
\text { Zeithalm (1988) }\end{array}$ \\
\hline & 39 & $\begin{array}{l}\text { Minha percepção de custo-benefício foi piorando ao longo do curso (Escala } \\
\text { Reversa, ajustada posteriormente na análise) }\end{array}$ & $\begin{array}{l}\text { Zielke (2010), } \\
\text { Zeithalm (1988) }\end{array}$ \\
\hline \multirow{2}{*}{$\begin{array}{ll}\text { Nível de } \\
\text { Preço }\end{array}$} & 1 & $\begin{array}{l}\text { Os preços dos cursos de pós-graduação geralmente são muito baixos na } \\
\text { INSTITUIÇÃO }\end{array}$ & Zielke (2010) \\
\hline & 3 & $\begin{array}{l}\text { Os preços dos cursos de pós-graduação na INSTITUIÇÃO são mais baratos } \\
\text { que em outras Instituições }\end{array}$ & Zielke (2010) \\
\hline
\end{tabular}

REAd | Porto Alegre - Edição 76 - N 3 - setembro/dezembro 2013 - p. 646-674 
A percepção da imagem de preço em serviços: um estudo de abordagem quantitativa sobre os cursos de lato sensu de uma universidade

\begin{tabular}{|c|c|c|c|}
\hline Construto & $\mathbf{N}^{\mathbf{o}}$ & Variáveis & Adaptado de: \\
\hline & 36 & $\begin{array}{l}\text { Levando-se em consideração o que é pago na pós-graduação, posso dizer } \\
\text { que a INSTITUIÇÃO tem um preço econômico (barato) }\end{array}$ & Zielke (2010) \\
\hline \multirow{3}{*}{$\begin{array}{l}\text { Dimensão } \\
\text { Emocional }\end{array}$} & 8 & É injusto continuar pagando por este curso após o término das disciplinas & $\begin{array}{l}\text { Zielke (2006, } \\
2011)\end{array}$ \\
\hline & 31 & O preço deste curso de pós-graduação me deixa insatisfeito & $\begin{array}{l}\text { Zielke }(2006, \\
\text { 2011) }\end{array}$ \\
\hline & 32 & O preço deste curso de pós-graduação me deixa irritado & $\begin{array}{l}\text { Zielke (2006, } \\
\text { 2011) }\end{array}$ \\
\hline
\end{tabular}

Fonte: Elaborado pelos autores, com base em Zielke (2006, 2010, 2011), Schuler et al. (2010)

A coleta das questões foi realizada em 20 turmas de cursos de lato sensu e MBA desta Universidade, selecionadas de forma probabilística, e o número de respondentes foi de 184 estudantes. Adotou-se como estratégia de coleta entregar os questionários e aguardar os alunos responderem, sendo que a aplicação levou, em média, 15 minutos por turma.

Os resultados foram tabulados no Microsoft Excel e para as análises quantitativas foi utilizado o PASW 18, de modo a efetuar a análise fatorial, alfa de Cronbach, regressão múltipla, análise de clusters e análise de variância.

Nesse sentido, para uma correta aplicação das técnicas de análise multivariada, os dados brutos foram tratados com as seguintes premissas: a) o estudo de casos específicos através dos dados perdidos ou valores ausentes (missing data ou missing values) e das observações atípicas (outliers); b) estudos relacionados à distribuição dos dados e relacionamento entre as variáveis realizados através das análises de normalidade, multicolinearidade, linearidade e homocedasticidade dos dados (Hair et al., 2009).

A extensão dos dados perdidos tem por objetivo determinar se os dados perdidos são suficientemente elevados para garantir um diagnóstico de aleatoriedade de perdas de dados. Segundo Hair Jr. et al. (2009), quando a quantidade de dados perdidos tanto para as variáveis quanto para os casos for abaixo de $10 \%$ e nenhum padrão não aleatório apareça, eles poderão ser ignorados. Analisando as variáveis do instrumento de pesquisa observou-se que as variáveis que compunham a escala de imagem de preço que mais apresentaram dados perdidos foram as variáveis 7 (a relação entre preço e desempenho é considerada pior na Instituição que em outras) com 18\% de não respostas e as variáveis 9 (a comparação de preço leva mais tempo aqui que em outras instituições com 38,95\% de não respostas) e a variável 23 (se eu avaliar os atributos da Instituição tal como módulo internacional, o preço é adequado) com $36,84 \%$ de casos perdidos. O que se observa é que a não resposta das questões 7 e 9 refere-se ao fato que os estudantes não terem condições de fazer comparativos uma vez que não utilizam os serviços das demais instituições. E com relação à questão 23 o elevado 
número de não respostas refere-se a fato que muitos cursos pós-graduação não possuem o módulo internacional. Com relação aos casos perdidos nos respondentes identificaram-se 28 casos com mais de $10 \%$ de não respostas, os quais foram excluídos da amostra. Com essa redução a amostra ficou então com 163 casos.

Submetendo a amostra ao teste de Little, identificou-se o seguinte: $\mathrm{x}^{2}=1986,093$; graus de liberdade $=1788$; significância de $\mathrm{p}=0,001$. Nesse sentido, identifica-se que os dados perdidos nesta pesquisa são perdidos ao acaso ou MAR (HAIR Jr. et al., 2009). Assim, a amostra recebeu o tratamento pela abordagem Expectation-Maximization - EM, através da utilização do software PASW 18, para a estimação e atribuição dos dados perdidos (HAIR Jr. et al., 2009).

$\mathrm{Na}$ detecção de observações atípicas (outliers) univariadas e multivariadas foram excluídos 4 casos por apresentarem valores padronizados superiores a |3|. Assim, com a exclusão destes 4 casos a amostra resultou em 159 casos $(\mathrm{n}=159)$, que foram então submetidos aos testes das suposições de análise multivariada, conforme é mostrado a seguir.

Pode-se atestar a normalidade a partir de métodos estatísticos univariados ou multivariados. Nesse sentido, foi utilizada a análise univariada considerando os valores de skewness e de kurtosis, além do teste de kurtosis multivariada de Mardia. Sendo que a amplitude dos valores de assimetria de Skewness compreendeu entre os -0,972 para o menor valor e 0,666 para o maior valor com uma média de -0,198. Quanto a amplitude de Kurtosis, o menor valor foi de $-1,39$ e 0,3 para o maior valor, ficando com uma média de -0,616. A partir do recomendado por Hau e Marsh (2004) identifica-se que os dados são moderadamente nãonormais. Na perspectiva da análise da normalidade multivariada a partir do teste de Mardia, observou-se que a estimativa da Kurtosis multivariada foi de 72,81 com uma razão crítica de 15,48. Quando estes valores estimados são altos eles indicam uma significante e positiva Kurtosis (BYRNE, 2009). Assim, a partir dos valores de normalidade de Mardia nesta magnitude parece evidente que os dados apresentam uma moderada normalidade multivariada.

Há um razoável consenso na literatura quanto às dificuldades de serem obtidos dados normais em estudos empíricos em ciências sociais (HAIR et al., 2009; BYRNE, 2009). Nesse sentido, quando os dados apresentam uma não normalidade adequada, há algumas medidas corretivas para o tratamento das amostras não normais. Um destes procedimentos é através da abordagem ML (maximum likelihood) com aplicação do processo de bootstrapping. 
A percepção da imagem de preço em serviços: um estudo de abordagem quantitativa sobre os cursos de lato sensu de uma universidade

Para o teste de homocedasticidade utilizou-se o teste $\mathrm{M}$ de Box e o teste de Levene, sendo que nenhuma variável apresentou padrões de heteroscedasticidade nas três variáveis não-métricas (sexo, idade e renda). O teste de linearidade consiste na análise da relação das variáveis com os padrões de associação entre cada par de variáveis e com a capacidade do coeficiente de correlação em representar adequadamente a relação. Analisando os coeficientes de Correlação de Pearson, é possível afirmar a existência de suposição de linearidade dos dados em todos construtos. Na análise da multicolinearidade através dos testes de Tolerância identificaram que todas variáveis apresentam multicolinearidade aceitável, ou seja tolerância entre 0,46 a 087 e Fator de Inflação da Variância (VIF) entre 1,35 a 2,09.

\section{RESULTADOS}

Em relação à amostra desta pesquisa, observa-se que dos 159 respondentes, 55\% são do sexo feminino e $45 \%$ do sexo masculino, sendo que $44 \%$ estão no início do curso, $18 \%$ no meio do curso e $38 \%$ estão no final do curso. Com relação à idade, 52\% têm até 30 anos, $31 \%$ de 31 a 40 anos e 17\% mais de 41 anos. Sobre a renda familiar dos entrevistados, $26 \%$ têm uma renda de até $\mathrm{R} \$ 2.000,00,31 \%$ de $\mathrm{R} \$ 2001,00$ à $\mathrm{R} \$ 4.000,00,30 \%$ de $\mathrm{R} \$ 4.001,00$ à $\mathrm{R} \$$ $8.000,00$ e $13 \%$ ganham mais de $\mathrm{R} \$ 8.000,00$.

Em síntese, esta amostra é composta por estudantes já inseridos no mercado de trabalho e de um bom poder aquisitivo, o que reflete a característica da população composta pelos estudantes de pós-graduação nesta Instituição, considerando a realidade da região de inserção da Universidade. A partir dela, desenvolveu-se nesta seção a análise fatorial visando à confirmação das dimensões de Imagem de Preço, e bem como a utilização de outras técnicas multivariadas visando analisar as relações deste fenômeno com a intenção de compra.

\subsection{Validação da Escala de Imagem de Preço em Serviços}

Para a identificação das dimensões da Imagem de Preço, buscou-se no primeiro momento efetuar o procedimento de análise Fatorial sobre os dados coletados, objetivando condensar as diversas variáveis em um número menor de fatores, de modo a identificar as dimensões e facilitar o processo de análise.

A primeira etapa consistiu em rodar esta análise com o suporte do aplicativo PASW 18, com extração pelo Método dos Componentes Principais, baseados nos autovalores; rotação Varimax e suprimindo-se os coeficientes abaixo de 0,4. Para esta primeira análise os

REAd | Porto Alegre - Edição 76 - N 3 - setembro/dezembro 2013 - p. 646-674 
indicadores da dimensão de recompra não foram incluídos no modelo por tratar-se de uma variável dependente.

Como resultado da análise fatorial final após os refinamentos realizados, obteve-se um índice KMO de 0,876 e significância no Teste de Bartlet, com 4 fatores que explicam $67 \%$ da variabilidade dos dados. Com base nos fatores, foi realizada a análise do alfa de Cronbach, de modo a verificar a consistência interna do grupo de variáveis. Neste sentido, apresenta-se na tabela 1 o resultado consolidados das dimensões, médias, desvio padrão e alfa de

\section{Cronbach.}

Os resultados da Analise Fatorial exploratória indicam uma boa consistência interna das variáveis propostas e dos constructos e também uma adequada explicação ao que o estudo se propõe a medir. Nesse sentido, a adaptação da escala de Zielke (2010) com a adequação do estudo de Schuler et al. (2010) foi adequada e tal instrumento pode servir de base orientativa para a mensuração da imagem de preço das IES de seus cursos de pós-graduação lato sensu.

Tabela 1 - Resultados da Análise Fatorial e do Alfa de Cronbach

\begin{tabular}{|c|c|c|c|c|c|c|c|c|}
\hline Fato & Dimensão & Média & DP & $\% \mathrm{Var}$ & Alfa & Itens & Descrição & Carga \\
\hline \multirow{6}{*}{1} & \multirow{6}{*}{$\begin{array}{l}\text { Dimensão } \\
\text { Funcional }\end{array}$} & \multirow{6}{*}{4,49} & \multirow{6}{*}{1,76} & \multirow{6}{*}{40,46} & \multirow{6}{*}{0,87} & Q22 & $\begin{array}{l}\text { Se eu avaliar os atributos da INSTITUIÇÃO, tal como a } \\
\text { biblioteca,o preço é adequado }\end{array}$ &, 749 \\
\hline & & & & & & Q25 & $\begin{array}{l}\text { Se eu avaliar a localização e a acessibilidade da } \\
\text { INSTITUIÇÃO, o preço é adequado }\end{array}$ & ,740 \\
\hline & & & & & & Q21 & $\begin{array}{l}\text { Se eu avaliar os atributos do curso de pós-graduação da } \\
\text { INSTITUIÇÃO, tal como o corpo-docente, o preço é } \\
\text { adequado }\end{array}$ & ,707 \\
\hline & & & & & & Q24 & $\begin{array}{l}\text { Se eu avaliar os atributos da INSTITUIÇÃO, tal como as } \\
\text { disciplinas oferecidas, o preço é adequado }\end{array}$ & 685 \\
\hline & & & & & & Q20 & $\begin{array}{l}\text { Se eu avaliar os atributos do curso de pós-graduação da } \\
\text { Instituição, tal como infraestrutura, o preço é adequado }\end{array}$ & ,683 \\
\hline & & & & & & Q19 & $\begin{array}{l}\text { Em relação ao nível de qualidade do curso de pós- } \\
\text { graduação da INSTITUIÇÃO, o preço bastante adequado }\end{array}$ &, 595 \\
\hline \multirow{5}{*}{2} & \multirow{5}{*}{$\begin{array}{c}\text { Valor } \\
\text { Percebido }\end{array}$} & \multirow{5}{*}{4,41} & \multirow{5}{*}{0,74} & \multirow{5}{*}{12,70} & \multirow{5}{*}{0,84} & Q39 & $\begin{array}{l}\text { Minha percepção de custo-benefício foi piorando ao } \\
\text { longo do curso }\end{array}$ & ,849 \\
\hline & & & & & & Q30 & $\begin{array}{l}\text { Minha percepção de custo-benefício piorou logo após o } \\
\text { início das aulas }\end{array}$ & ,781 \\
\hline & & & & & & Q29 & $\begin{array}{l}\text { Minha percepção de custo-benefício era melhor antes do } \\
\text { início das aulas }\end{array}$ & ,772 \\
\hline & & & & & & Q6 & $\begin{array}{l}\text { Comparada a outras instituições, o custo-benefício é } \\
\text { muito bom na Instituição }\end{array}$ & ,458 \\
\hline & & & & & & Q5 & Este curso de pós-graduação vale o que eu pago por ele & ,462 \\
\hline \multirow{3}{*}{3} & \multirow{3}{*}{$\begin{array}{l}\text { Nível de } \\
\text { Preço }\end{array}$} & \multirow{3}{*}{3,40} & \multirow{3}{*}{1,25} & \multirow{3}{*}{8,01} & \multirow{3}{*}{0,75} & Q3 & $\begin{array}{l}\text { Preços dos cursos de pós-graduação na INSTITUIÇÃO } \\
\text { são mais baratos que em outras Instituições }\end{array}$ & ,836 \\
\hline & & & & & & Q1 & $\begin{array}{l}\text { Preços dos cursos de pós-graduação geralmente são } \\
\text { muito baixos na INSTITUIÇÃO }\end{array}$ & ,768 \\
\hline & & & & & & Q36 & $\begin{array}{l}\text { Levando-se em consideração o que é pago na pós- } \\
\text { graduação, posso dizer que a INSTITUIÇÃO tem um } \\
\text { preço econômico (barato) }\end{array}$ & ,709 \\
\hline
\end{tabular}

REAd | Porto Alegre - Edição 76 - N 3 - setembro/dezembro 2013 - p. 646-674 
A percepção da imagem de preço em serviços: um estudo de abordagem quantitativa sobre os cursos de lato sensu de uma universidade

\begin{tabular}{l|l|l|l|l|l|l|l|l}
\hline & & & & & Q8 & $\begin{array}{l}\text { É injusto continuar pagando por este curso após o } \\
\text { término das disciplinas }\end{array}$ &, 762 \\
\cline { 5 - 7 } & $\begin{array}{l}\text { Dimensão } \\
\text { Emocional }\end{array}$ & 3,74 & 1,52 & 6,07 & 0,71 & $\mathrm{Q} 32$ & O preço deste curso de pós-graduação me deixa irritado &, 691 \\
\cline { 5 - 7 } & $\mathrm{Q} 31$ & $\begin{array}{l}\text { O preço deste curso de pós-graduação me deixa } \\
\text { insatisfeito }\end{array}$ &, 663 \\
\hline
\end{tabular}

Fonte: Elaborado pelos autores, com base nos resultados do PASW.

\subsection{A relação entre as dimensões da Imagem de Preço e a etapa do curso}

A partir dos resultados da análise fatorial confirmatória, foi realizada a análise da variância (ANOVA) em relação às dimensões como variáveis independentes e o estágio do aluno no curso como variável dependente, sendo apresentado na tabela 2. Considerando que a experiência de consumo varia ao longo do período de um curso, considerou-se importante analisar se há mudança na percepção de imagem de preço durante o início, meio e final do curso..

Nesta mesma análise foi realizado um post hoc com o teste de Tukey, para avaliar a diferença entre cada grupo, sendo que os valores em cinza representam diferenças não significativas.

Tabela 2 - Resultados da ANOVA

\begin{tabular}{c|c|c|c|c}
\hline Dimensão & Final & Meio & Inicio & Sig \\
\hline Funcional & 4,1996 & 4,4260 & 4,7248 &, 0449 \\
\hline Valor Percebido & 4,0628 & 4,2306 & 4,2674 &, 3234 \\
\hline Nível de Preço & 3,1008 & 3,4467 & 3,7539 &, 0180 \\
\hline Emocional & 3,3978 & 3,5641 & 4,1933 &, 0161 \\
\hline Intenção de Recompra & 3,7657 & 4,0905 & 4,5379 &, 0071 \\
\hline
\end{tabular}

Fonte: Elaborado pelos autores, com base nos resultados do PASW.

A partir da análise da variância (ANOVA) observou-se que há diferença significativa nas dimensões funcional e emocional entre todos os estágios. Não se verificou variação significativa entre os estágios inicio e meio do curso nas dimensões Valor Percebido e Nível de Preço, apesar desta diferença existir entre o meio e o final do curso.

No que se refere às diferenças significativas (sig. 0,0449) na dimensão funcional, observa-se que uma imagem mais forte da utilidade percebida nesta dimensão no que tange aos seus propósitos funcionais, utilitários e físicos tal como é apresentado por Sheth et al. (1991) está mais presente na percepção de estudantes no início do curso que no final. Tal manifestação pode ser coerente, uma vez que estudantes no final do curso tendem a ficar mais 
familiarizados com a infraestrutura e também mais exigentes com relação ao corpo docente e a qualidade do curso. Um vez que tais estudantes possuem mais experiências com relação à instituição, tornam-se também mais críticos e sugestivos com relação a sua satisfação no que se refere aos aspectos funcionais.

Segundo Sheth et al. (1991) as emoções impactam nas decisões de escolha, dessa forma, emoções negativas podem impactar tanto num boca a boca negativo quanto num sentimento de injustiça com relação ao preço cobrado. O que chama a atenção no que tange a diferença significativa (sig. 0,016) na dimensão emocional é que os estudantes no início do curso estão mais propensos à emoções negativas com relação ao preço cobrado do que os estudantes no fim do curso. Isso de certa forma pode ser considerado algo positivo, pois identifica-se que ao longo do tempo os estudantes vão percebendo mais valor e justiça no preço cobrado. Assim, como afirma Peine et al. (2009), os consumidores que percebem uma oferta alvo com mais valor e justa também apresentam maior desejo de comentar palavras positivas, o que favorece a construção de uma imagem mais favorável dos cursos.

Para Siems et al. (2008) os efeitos da satisfação do preço possuem uma relação positiva com a satisfação geral do consumidor e consequentemente na lealdade do consumidor. No entanto observa-se uma diferença significativa (sig. 0,007) entre o estágio que o estudante está no curso e intenção de recompra (média do bloco respectivo de questões). Assim, os estudantes no início do curso têm uma maior predisposição para recompra (média 4,54 em uma escala de 7 pontos) que os estudantes do final do curso (média $3,77)$. Esse resultado pode ser visto inicialmente como algo negativo, mas que no entanto merece ser analisado com maior profundidade, uma vez que os estudantes ao final do curso, além de terem estado mais em contato com o serviço, podem ter diferentes ambições ou planos para o futuro, que pode não estar incluso o de voltar a fazer um outro curso de pósgraduação por exemplo. Contudo, observa-se que os alunos que estão no meio do curso têm uma intenção de recompra significativamente menor que a dos os alunos de inicio de curso e intenção de recompra significativamente maior que as dos alunos de final de curso, identificando, neste caso, uma relação inversa entre o período do curso e a intenção de recompra.

Para fortalecer este argumento, foi utilizada a Análise de Cluster, a partir do método Two-Step Cluster, uma vez que trabalha com variáveis tanto categóricas quanto contínuas, e tem condições de localizar um número de clusters automaticamente. Evidencia-se que de acordo Nagle e Holden (2003), é importante identificar estes segmentos de mercado, pois os

REAd | Porto Alegre - Edição 76 - N 3 - setembro/dezembro 2013 - p. 646-674 
A percepção da imagem de preço em serviços: um estudo de abordagem quantitativa sobre os cursos de lato sensu de uma universidade

mesmos possuem diferentes percepções e sensibilidade ao preço, e esta técnica multivariada possibilita esta análise.

Foram utilizadas como variáveis contínuas a Dimensão Funcional e o Valor Percebido, pois foram as que se mantiveram no modelo de regressão, apresentado na seção 4.3. No entanto, como critério de separação foi determinado um número de três grupos, visando identificar um nível alto, médio e baixo de percepção de Imagem de Preço. Como resultado da análise, verificou-se que houve um bom nível de coesão e separação, de acordo com os indicadores fornecidos pelo output do PASW (Cluster quality: silhouete measure of cohesion and separation). Considerando-se a relação entre o momento do curso e os clusters, observase na tabela 3 a seguinte proporcionalidade em relação às categorias (início, meio, final).

Tabela 3 - Percentual de respondentes de cada etapa do curso nos clusters

\begin{tabular}{c|c|c|c}
\hline $\begin{array}{l}\text { Variável } \\
\text { Etapa do Curso }\end{array}$ & $\begin{array}{c}\text { Cluster 1 } \\
\text { Baixo Nível }\end{array}$ & $\begin{array}{c}\text { Cluster 2 } \\
\text { Médio Nível }\end{array}$ & $\begin{array}{c}\text { Cluster 3 } \\
\text { Alto Nível }\end{array}$ \\
\hline Final & $55 \%$ & $31 \%$ & $20 \%$ \\
\hline Meio & $19 \%$ & $16 \%$ & $17 \%$ \\
\hline Início & $26 \%$ & $53 \%$ & $63 \%$ \\
\hline
\end{tabular}

Fonte: Elaborado pelos autores, com base nos resultados do PASW.

Neste caso, verifica-se que há uma predominância dos alunos de final de curso no Cluster $1(63 \%)$ e uma maior predominância dos alunos de inicio de curso no Cluster 3 (55\%), indicando uma mudança na imagem de preço em função da etapa do curso. Uma vez que há diferentes experiências nestas etapas, tanto em relação ao acesso do aluno às dimensões funcionais, e que a percepção de valor percebido vai se construindo na medida em que o aluno avança nos estudos e percebe de forma gradativa o retorno do investimento, essa diferença pode ser esperada (SHETH et al., 1991).

\subsection{A relação entre a Imagem de Preço e Intenção de Recompra}

A partir da análise de regressão múltipla verificou-se a influência das variáveis independentes (fatores) sobre a variável dependente intenção de recompra, formado pelos itens $12,13,15$ e 17, elaborados conforme a escala proposta por Zielke (2010). Por tratar-se de uma variável dependente, estas questões não foram incluídas na primeira fatorial com os demais itens. Entretanto, foi utilizada a analise fatorial para estes quatro itens isoladamente e se observou a existência de somente um fator, indicando que há correlação entre eles. $\mathrm{O}$ 
Alpha de Cronbach destes itens é de 0,823, sendo que a média da Intenção de Compra foi de 4,19, com um desvio padrão de 1,34.

Como resultado da análise de regressão utilizando-se o método stepwise, obteve-se um modelo com $\mathrm{R}^{2}$ de 0.494 , sendo que as dimensões apresentaram um impacto mais significativo sobre a intenção de recompra foram a Funcional $(\beta=0,488, p=0,000)$ e Valor $(\beta=0,406, p=0,000)$, e as dimensões de Nível de Preço e Emocional não apresentaram significância inferior a 0,05 .

Com este modelo, observa-se uma relação de custo x benefício, ou seja, o que mais influencia na percepção de escolher cursos de pós no futuro ou de escolher a mesma IES no futuro (intenção de recompra) tem relação mais forte com a Dimensão Funcional, ou os atributos atuais que o curso disponibiliza em relação ao preço pago, e também em relação ao Valor percebido. Neste caso, trata-se de um julgamento de valor e justiça mais amplo que apenas em relação aos atributos. Assim, como é identificado por Lichtenstein et al. (1993), a dimensão funcional como dica de qualidade, e a dimenão de valor percebido como dica de benefício esperado a partir do sacrifício realizado estão positivamente relacionadas.

Pode ser considerado que pesem mais estas dimensões pelo consumidor compreender e avaliar com maior profundidade a sua experiência na percepção de valor, conforme exposto por De Toni (2005). Como a pesquisa foi aplicada com alunos que estão cursando o curso, há agora um conhecimento de causa, haja visto que a sua imagem de preço é afetada pelo o seu tempo de curso (conforme observada na ANOVA), e não se trata mais uma de percepção advinda de uma imagem de marca ou de processos de ancoragem, como por exemplo, em função da comparação de diferentes modelos de cursos a partir de uma entrevista com os coordenadores. Por esta razão, pode se atribuir que a variável nivel de preço não tenha entrado no modelo. Poderia se supor que esta variável é importante para a intenção de compra no primeiro momento (antes de cursar o curso), mas após a experiência já ter ocorrido, outras variáveis passam a predominar sobre a intenção de recompra. Este resultado se traduz em estratégias de comunicação da precificação, em termos da oferta para alunos que estão cursando e em relação à novos alunos.

Baseando-se ainda na Análise de Variância (ANOVA), dos clusters de percepção de Imagem de Preço previamente identificados em relação à variável intenção de recompra, observa-se uma relação positiva. Neste caso, verifica-se que há diferença significativa entre os clusters em relação à intenção de recompra, sendo que essa variação ocorre entre os grupos, conforme observado pelo teste de Tukey apresentado na tabela 7 .

REAd | Porto Alegre - Edição 76 - N 3 - setembro/dezembro 2013 - p. 646-674 
A percepção da imagem de preço em serviços: um estudo de abordagem quantitativa sobre os cursos de lato sensu de uma universidade

Tabela 7 - Resultados do Teste de Tukey

\begin{tabular}{c|c|c|c|c}
\hline Cluster (I) & Média (J) & $\begin{array}{c}\text { Cluster } \\
\text { Comparado }\end{array}$ & $\begin{array}{c}\text { Diferença de } \\
\text { média (I-J) }\end{array}$ & Sig. \\
\hline 1 & 3,22 & 2 & 1,10 & 0,00 \\
\hline 2 & & 3 & 2,24 & 0,00 \\
\hline & 4,37 & 1 & $-1,10$ & 0,00 \\
\hline 3 & & 3 & 1,14 & 0,00 \\
\hline & 5,47 & 1 & $-2,24$ & 0,00 \\
\hline & & 2 & $-1,14$ & 0,00 \\
\hline
\end{tabular}

Fonte: Elaborado pelos autores, com base nos resultados do PASW.

Neste sentido, verifica-se que o nível de recompra é maior no cluster que possui maior nível de imagem de preço, neste caso, envolvendo a Dimensão Funcional e o Valor Percebido, ou seja, reforçando a relação positiva entre o a Imagem de Preço e a Intenção de Compra, conforme abordado por Siems et al. (2008).

Tendo em vista as análises de regressão, de cluster e de variância, observa-se portanto que os alunos que possuem uma percepção de imagem de preço mais positiva em relação aos cursos apresentaram uma maior intenção de recompra, que por sua vez é influenciada pela dimensão funcional e pelo valor percebido.

\section{CONSIDERAÇÕES FINAIS}

Imagem de preço não é uma simples tradução do preço atual cobrado pela empresa, mas sim uma função de vários aspectos, os quais envolvem processos subjetivos, como os associados ao preço percebido e à qualidade dos serviços prestados (ZIELKE, 2010). Por outro lado, é pertinente que as Universidades e Faculdades desenvolvam diferenciais em suas ofertas, assim como na forma como elas são vendidas e sua imagem percebida pelo mercado. Assim, o objetivo deste trabalho foi identificar a percepção da imagem de preço dos alunos dos cursos de lato sensu de uma Universidade e a sua relação com a intenção de recompra.

Dentre as contribuições desta pesquisa podem-se destacar três. Primeiramente o estudo de imagem de preço de serviço é um tema pouco explorado. Zielke (2006, 2010 e 2011) é um dos poucos pesquisadores que abordam está temática, e cuja pesquisa está concentrada na imagem de preço de lojas. Neste panorama, não se identificaram estudos relacionados à imagem de preço de serviço e mais especificamente a serviços de cursos de pós-graduação. Nesse sentido, este estudo auxilia a melhor entender como as imagens de preço de serviço podem ser configuradas e seus impactos sobre a intenção de compra de um produto ou 
serviço, uma vez que Siems et al. (2008) apontam que o constructo percepção de preço como um influenciador da satisfação e da lealdade.

Num segundo momento identificou-se que a imagem de preço de serviço pode ser formada a partir das dimensões Funcional, do Valor Percebido, do Nível de Preço e a Emocional. Neste sentido, este estudo contribui na perspectiva teórica na medida em que adapta o estudo proposto por Zielke (2010) e Schuler et al. (2010), num contexto de serviços. Os resultados da pesquisa identificam que $67 \%$ da variância pode ser explicado a partir destas quatro dimensões. Portanto estes achados são importantes uma vez que complementam os estudos realizados com preço de varejo adaptados ao contexto de preço de serviço, bem como evidenciam dimensões como o papel do emocional, conforme abordado por abordado por Sheth et al. (1991), e a sua relação com a Imagem de Preço.

Uma terceira contribuição foi a proposição de uma escala de medida para analisar cada um dos construtos propostos. Tanto a academia quanto a comunidade gerencial carecem de um instrumento para a mensuração da imagem de preço de serviço de ensino e sua relação com a intenção de recompra dos consumidores. Os achados identificam que as dimensões funcionais e de valor percebido são as que mais impactam na intenção de recompra e que o conjunto das quatro dimensões influenciam aproximadamente $50 \%$ em suas escolhas.

Sob a luz destes resultados, observa-se que o desenvolvimento de novos estudos visando maior compreensão sobre a Teoria da Imagem de Preço pode auxiliar as organizações a desenvolverem vantagem competitiva, a partir da definição de estratégias de precificação que estejam fundamentadas neste fenômeno. Ainda, uma vez que a intenção de recompra constitui-se numa forma de lealdade (FORNELL et al, 1996) e o preço é um atributo significativo, é que compreender a imagem de preço que o cliente tem da organização torna-se uma atividade importante para que ela potencialize seus resultados financeiros. Tendo em vista que observou-se que há relação positiva entre a Imagem de Preço e a intenção de recompra, se fortalece a teoria de Lichtenstein et al., (1993) em termos de relação preço e probabilidade de compra, agregando-se as demais dimensões nesta compreensão do constructo preço, no caso de serviços.

Todavia, evidencia-se que esta teoria necessita ainda de maiores pesquisas, uma vez que se observou diferença entre o estudo da Imagem no contexto do varejo e de serviços, o que levanta a hipótese de que novas dimensões possam surgir em outros contextos, e inclusive com novas diferenciações no contexto de serviço. Observa-se aqui, por exemplo, a imagem de preço no contexto de business-to-business (cuja dinâmica de compra difere do business-to- 
A percepção da imagem de preço em serviços: um estudo de abordagem quantitativa sobre os cursos de lato sensu de uma universidade

client) e com comportamento diferente também para o caso da graduação, onde devido a um tempo maior de curso, com um nível diferente de expectativas, tendo em vista que se trata da primeira experiência de formação de ensino superior do aluno, tem-se a hipótese de que a dimensão emocional possa ser mais expressiva que no caso de cursos de lato sensu, cujo publico já é formado por profissionais da área.

Desta maneira, sugere-se para futuros estudos que o contexto da pesquisa seja tratado como uma variável moderadora, uma vez que se observou diferença neste fenômeno tanto no contexto de estudo de Zielke (2010), varejo, quanto no contexto nesta pesquisa, serviços educacionais de pós-graduação lato sensu. Para o caso de serviços, é imprescindível a adoção e a avaliação do tempo de experiência, que se demonstrou uma variável com diferenças significativas.

Sob o ponto de vista gerencial, este estudo contribui na medida em que apresenta que os aspectos funcionais e de percepção de custo-benefício (valor percebido) influenciam na intenção de recompra, neste contexto de aplicação. Com essa informação, é possível que a Coordenação de um curso tenha mais subsídios para elaborar estratégias de satisfação e de fidelização para seus alunos, bem como de analisar que há necessidade de se observar de modo distinto alunos que estão no início e no final do curso, uma vez que sua percepção de imagem de preço difere.

Entretanto, este estudo limita-se por não investigar esta imagem de preço em relação a alunos que ainda não ingressaram num curso de lato sensu, e bem como a comparação desta imagem entre IES concorrentes. Uma hipótese é que havendo diferença entre os modelos da Imagem de Preço, isso implicaria, em termos gerenciais, a necessidade de duas posturas distintas, mas complementares, de valorizar diferentes atributos - quando se refere à comunicação e imagem transmitida, para o seu público interno e externo. Mas esta lacuna, entretanto, reflete-se numa agenda de futuras pesquisas, de modo a fortalecer o modelo de Imagem de Preço.

\section{REFERÊNCIAS}

ARIELY, Dan. Previsivelmente irracional: as forças ocultas que formam as nossas decisões. Rio de Janeiro: Elsevier, 2008. 
AVLONITIS, G. G., INDOUNAS, K. A. Pricing practices of service organizations. Journal of Service Marketing, v. 20, n. 5, p. 346-356, 2006.

BARICH, H.: KOTLER, P. A framework for marketing image management. Sloan Management Review. Cambridge, v. 32, n. 2, p. 94-104, Winter 1991.

BERNARDI, L. A. Política e formação de preços: uma abordagem competitiva, sistêmica e integrada. São Paulo: Atlas, 1996.

BOLTON, Lisa E.; WARLOP, Luk; ALBA, Joseph W. Consumer Perceptions of Price (Un)Fairness. Journal of Consumer Research, Vol. 29, March, p. 474-491, 2003.

BOLTON, Ruth N.; LEMON, Katherine N.; VERHOEF, Peter C. Expanding Business-toBusiness Customer Relationships: Modeling the Customer's Upgrade Decision. Journal of Marketing, v. 72, p. 46-64, January 2008.

CAMPO, Sara; YAGÜE, María J. The perception of price discounts according to consumers characteristics. Journal of Revenue and Pricing Management, v. 6, n. 2 p. 86-99, 2007.

CHERNEV, A. Articulation Compatibility in Eliciting Price Bids. Journal of Consumer Research, v. 33, p. 329-341, December 2006.

COATS, R. Morris. Peak-Load Pricing and Facility Utilization in Higher Education. Journal of Marketing for Higher Education. Vol. 6, Iss. 2, 1995.

DE TONI, D. Administração da imagem de produtos: desenvolvendo um instrumento para a configuração da imagem de produto. Tese (Doutorado) - UFRGS - Universidade Federal do Rio Grande do Sul, Programa de Pós-Graduação em Administração, Porto Alegre, 2005.

DEMORANVILLE ,Carol W., O'DONNELL, Paula Bogott. Price Elasticity of Per-CreditHour Tuition Charges and the Effects on Four-Year Graduation Rates. Journal of Marketing for Higher Education. Vol. 11, Iss. 4, 2001 
A percepção da imagem de preço em serviços: um estudo de abordagem quantitativa sobre os cursos de lato sensu de uma universidade

DOBNI, D.; ZINKHAN, G. M. In search of grand image: a foundation analysis. Advances in Consumer Research, v. 17, n. 1, p. 110-119, 1990.

DOVALIENE, A.; VIRVILAITE, R. Customer value and its contribution to the longevity of relationship with service provider: the case of theatre industry. Iminerine EkonomikaEngineering economic, n.1, p. 66-73, 2008.

FORNELL, C., Johnson, M.D., ANDERSON, E.W., Cha, J. and Bryant, B.E. The American Customer Satisfaction Index: Nature, Purpose, and Findings, in: Journal of Marketing, v. 60, n. 4, p. 7-18, 1996.

GOURVILLE, J. T.; SOMAN, D. Pricing and the psychology of consumption. Harvard Business Review, v. 80, n. 9, p. 90-96, 2002.

HAIR JR., Joseph F. Fundamentos de métodos de pesquisa em administração. Porto Alegre, RS: Bookman, 2009.

HAMILTON, R.; CHERNEV, A. The impact of product line extensions and consumer goals on theformation of price image. Journal of Marketing Research, v. XLVII, p. 51-62, February 2010.

JANISESWKI, Chris. LICHTENSTEIN, Donald. A range theory account of price perception. Journal of Consumer Research. Vol. 25. March 1999.

KEARNEY, Estudo A. T. O que o consumidor valoriza? HSM Management, n.44 maio-Junho 2004.

KOTLER, P.; KELlER, K. L. Administração de marketing. 12. ed. São Paulo: Prentice Hall, 2006.

LARENTIS, Fabiano; GASTAL, Fernanda. O Estado da Arte do Conceito de Valor para o Cliente: Passado, Presente e Futuro. Encontro de Marketing da Anpad. 2004. 
LICHTENSTEIN, Donald R. RIDGWAY, Nancy M.; NETEMEYER, Richard G. Price perception and consumer shopping behavior: a field study. Journal of Marketing Research, 30, p. 234-245, 1993.

LITTLE, Michael W., O'TOOLE, Dennis, WETZEL James.The Price Differential's Impact on Retention, Recruitment, and Quality in a Public University. Journal of Marketing for Higher Education. Vol. 8, Iss. 2, 1997

MAYER V. F.; MARIANO, S.R. H; ANDRADE, C. L. T. Percepção de Preço e Valor no Mercado de Distribuição de Energia Elétrica: Proposta de um Modelo Conceitual. In: ENCONTRO ANUAL DA ANPAD, 33., 2009, São Paulo. Anais ... São Paulo: ANPAD, 2009.

MONROE, K. B. Pricing Making Profitable Decisions. 3. ed. Mc Graw-Hill Irwin, 2003.

MONROE, K. B. Pricing Making Profitable Decisions. 2rd ed. McGraw-Hill, New York, 1990.

MUNNUKKA, Juha. Pricing method as a tool for improved price perception. Journal of Revenue and Pricing Management, v.5, n. 3, p. 207-220, 2006.

NAGLE, T.; HOLDEN, R. K. Estratégias e táticas de preços: um guia para as decisões lucrativas. São Paulo: Prentice Hall, 2003.

ODUOZA. Chike F. Reflections on costing, pricing and income measurement at UK higher education institutions. Journal of Higher Education Policy and Management. Vol. 31, Iss. 2, 2009

PAIXÃO, Roberto B.; BRUNI, Adriano L.; SILVA, Sérgio C. M. Melhor e mais caro: um estudo sobre a associação entre a percepção dos preços e a qualidade dos produtos e serviços. Revista de Gestão USP, São Paulo, v. 13, n. 4, p. 39-50, out./dez. 2006.

PEINE, Kllaus; HEITMANN, Mark; HERRMANN, Andreas. Getting a Feel for Price Affect: A Conceptual Framework and Empirical Investigation of Consumers? Emotional Responses to Price Information. Psychology \& Marketing, v. 26, n.1, p. 39-66, January 2009. 
A percepção da imagem de preço em serviços: um estudo de abordagem quantitativa sobre os cursos de lato sensu de uma universidade

POIESZ, B. C. The image concept: its place in consumer psychology. Journal of Economic Psychology, v. 10, n. 4, p. 457-472, 1989.

QUIGLEY, Charles J., BINGHAM, Frank G., NOTARANTONIO, Elaine M., MURRAY, Keith. The Impact Discounts and the Price-Quality Effect Have on the Choice of an Institution of Higher Education. Journal of Marketing for Higher Education. Vol. 9, Iss. 2, 2000 .

SCHULER, M.; DE TONI, D.; MILAN, G. S.; PANIZZON, M.; LARENTIS, F. As Dimensões da Imagem de Preço para o Consumidor: Uma Abordagem Exploratória. In: ENCONTRO ANUAL DA ANPAD, 34., 2010, Rio de Janeiro. Anais... Rio de Janeiro: ANPAD, 2010.

SCHULER, Maria. A cultura organizacional como manifestação da multidimensionalidade humana. In: KUNSCH, M. M. K. (org.). Comunicação organizacional: linguagem, gestão e perspectivas. Volume 2. São Paulo: Saraiva, 2009. cap. 10, p. 243-274.

SHETH, J.N.; NEWMAN, B. I.; GROSS, B. L. Consumption values and market choice: theory and applications. Ohio: South Western Publishing, 1991.

SHAPIRO, Benson.The psychology of pricing. Harvard Business Review, 1968.

STERN, B.; ZINKHAN, G. M.; JAJU, A. Marketing images: construct definition, measurement issue, and theory development. Marketing Theory, v. 1, n. 2, p. 201-224, 2001.

TSIROS, Michael; HARDESTY, David M. Ending a price promotion: retracting It in one step or phasing it out gradually. Journal of Marketing, v. 74, p. 49-64, January 2010.

VIEIRA, Valer Afonso. MATOS, César Augusto. A influência da apresentação do preço sobre as percepções dos clientes. Revista de Administração Contemporânea. V 16. Art 4.

VIRVILAITE, Regina; SALADIENE, Violeta; SKINDARAS, Dalius. The Relationship between Price and Loyalty in Services Industry. Inzinerine Ekonomika-Engineering Economics, v.3, 2009. 
WERTENBROCH, K.; SOMAN, D.; CHATTOPADHYAY, A. On the Perceived Value of Money: The Reference Dependence of Currency Numerosity Effects. Journal of Consumer Research, v. 34, p.1-10, june 2007.

WINER, Russell. A Price Vector Model of Demand for Consumer Durables: Preliminary Developments. Marketing Science, v.4 , p.74-90, 1985.

ZEITHAML, Valarie A. Consumer Perceptions of Price, Quality, and Value: A Means-End Model and Synthesis of Evidence. Journal of Marketing, v. 52, p. 2-22, July 1988.

ZIELKE, Stephan. How price image dimensions influence shipping intentions for different store formats. In. European Journal of Marketing, v. 44, n. 6, p. 748-770, 2010.

ZIELKE, Stephan. Measurement of Retailers' Price Images with a Multiple-item Scale. Int. Rev. of Retail, Distribution and Consumer Research, v. 16, n. 3, p.297-316, July 2006.

ZIELKE, Stephan; TOPOROWSKI, Waldemar. Negative price-image effects of appealing store architecture: Do they really exist?. Journal of Retailing and Consumer Services. Volume 19, Issue 5, September 2012, Pages 510-518. 